\title{
Overall Media Use and ADHD-Related Behaviors Among Children in China: A Meta-Analysis
}

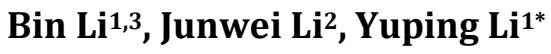 \\ ${ }^{1}$ Department of Communication and Media, Guangzhou Huashang College, Guangzhou 511300, China \\ ${ }^{2}$ Faculty of Science, The University of Auckland, Auckland 1142, New Zealand \\ ${ }^{3}$ College of Humanities, Zhuhai City Polytechnic, Zhuhai 519090, China \\ *Corresponding author: Yuping Li, shenx13393@163.com
}

\begin{abstract}
There are several theoretical reasons to believe that overall media use might be related to attention deficit hyperactivity disorder (ADHD) or ADHD-related behaviors (i.e., attention problems, hyperactivity, and impulsivity). Although studies on media-ADHD relationship have accumulated, they have yielded inconsistent results, especially those from different countries. Therefore, it is still undisclosed whether children's overall media use and ADHD-related behaviors are related to region and culture. A meta-analysis has been performed on three empirical studies investigating the relationship between overall media use and ADHD-related behaviors in children and adolescents from China. The results indicated significant relationship between overall media use and ADHD-related behaviors, $\mathrm{OR}=2.597$.
\end{abstract}

Keywords: ADHD; Media use; China; Child ADHD symptoms

Publication date: December 2021; Online publication: December 27, 2021

\section{Introduction}

Attention deficit hyperactivity disorder (ADHD) is one of the most common childhood behavioral disorders. China and western countries have different prevalence rates, ranging from $2.18 \%$ to $6.4 \%$ in China and $5 \%$ to $6 \%$ in western countries ${ }^{[1-3]}$. Based on the American Psychiatric Association, ADHD has been traditionally viewed as a discrete disorder, characterized by extreme levels of inattentiveness, hyperactivity, and impulsivity.

So far, the cause of ADHD is still uncertain, but it is generally believed to be associated with genetic, social, family, nutritional diet, and prenatal dates ${ }^{[4]}$.

Studies have shown that in recent years, the incidence of attention deficit hyperactivity disorder (ADHD) in China is increasing year by year ${ }^{[1]}$.

With the rapid development of electronic media, such as television, mobile phone, and tablet computer, the time spent on media among children is also increasing ${ }^{[5]}$.

Overexposure to electronic media has a significant impact on children, affecting their language learning ability and leading to attention deficit as well as various negative effects, such as aggression, eating disorders, sleep problems, and learning difficulties ${ }^{[6-8]}$. There have been recurrent claims in literatures that specific features of entertainment media may cause ADHD-related behaviors among children ${ }^{[9]}$. Several scholars have attributed the potential effects of entertainment media on ADHD-related behaviors to their violent nature ${ }^{[10-12]}$.

However, Chinese scholars have not been paying much attention to the relationship between media use and ADHD; therefore, a number of cross-section studies have been employed to evaluate the associations 
between overall media use and childhood ADHD.

This study sought to achieve the goal of assessing the association between overall media use and children's ADHD-related behaviors in China through a meta-analysis.

\section{Methods}

\subsection{Literature search}

This meta-analysis was designed to follow the principles of Preferred Reporting Items for Systematic Reviews and Meta-Analyses (PRISMA). Relevant studies were obtained using a two-step approach.

First, China Knowledge Resource Integrated (CNKI) databases were searched back to September 28, 2016, for studies on the relationship between overall media use and ADHD in children from among Chinese literatures. The following keywords were used to search for related studies by two independent investigators: "TV or television or game or hyperactive or impulsive" (in Chinese) and "ADHD or attention" (in Chinese). Second, the reference sections of the studies related to overall media use and ADHD-related behaviors were searched for additional citations.

The studies had to meet three criteria to be included in this meta-analysis.

First, they required the inclusion of overall media use. Overall media use refers to the overall amount of time spent viewing television (including movies) or playing video games on any platform (e.g., in hours per day).

Second, the studies had to be cross-sectional studies with a measure of ADHD-related behaviors. The included studies consisted of samples that compared children who have been clinically diagnosed with ADHD with those who do not have ADHD (control group).

Third, only studies with child participants younger than 18 years of age were included in this metaanalysis.

Figure 1 shows the results of literature search and selection. A total of 453 articles were identified from CNKI.

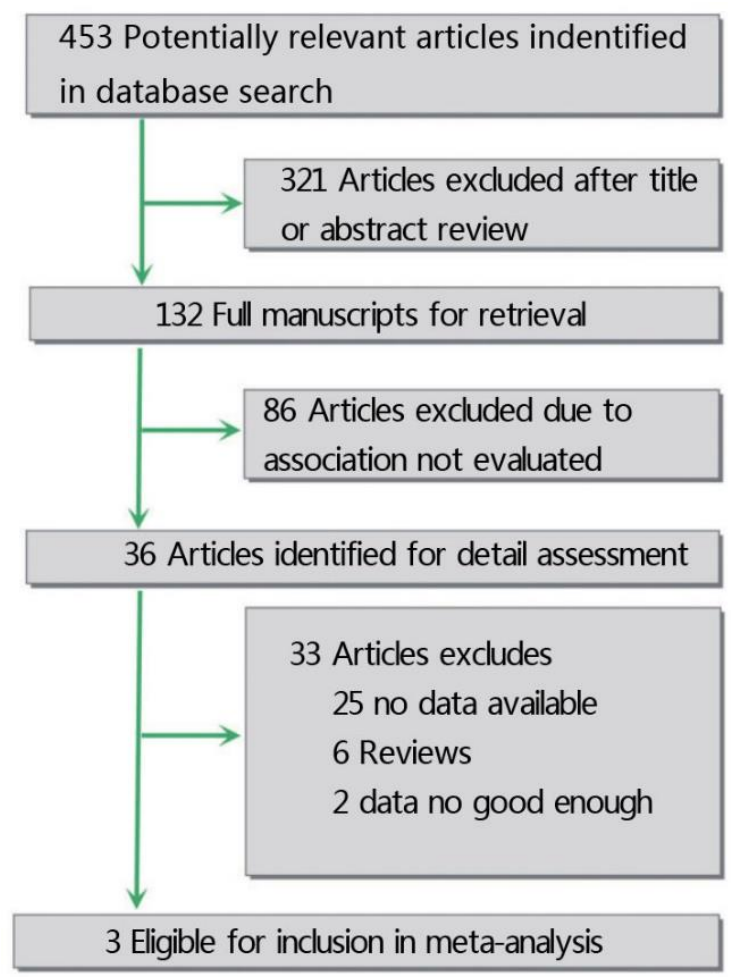

Figure 1. Flowchart of articles identified 
After the exclusion of duplicates and studies that did not fulfill the inclusion criteria, out of the five studies that met the aforementioned three inclusion criteria, two studies were excluded because the effect sizes could not be computed due to missing statistics ${ }^{[13,14]}$; therefore, three articles with a total of 632 subjects were included in this meta-analysis.

For the outcome of interest, pooled estimates and 95\% confidence intervals for effect sizes (OR) were calculated by using the Mantel-Haenszel weighted fixed effect meta-analysis. The $\mathrm{I}^{2}$ statistic was used to assess heterogeneity among studies; $\mathrm{I}^{2}$ values of $0 \%, 25 \%, 50 \%$, and $75 \%$ represent no, low, moderate, and high heterogeneity, respectively.

\subsection{Statistical analysis}

The following data were extracted from each study: first author's surname, publication year, study location, study size, overall media use categories, and adjustment factors in the multivariable analysis. The OR and 95\% CI for each study were calculated from the original data. For those studies with several media use categories, they were combined according to one-hour media use, which is over or below one-hour media use each day.

All analyses were performed using STATA 12.0 (Stata Corp, College Station, TX, USA). A two-sided $\mathrm{P}$ value $<0.05$ is considered statistically significant.

\section{Results}

The OR for each study and the combined OR for the overall media use categories are shown in Figure 2.

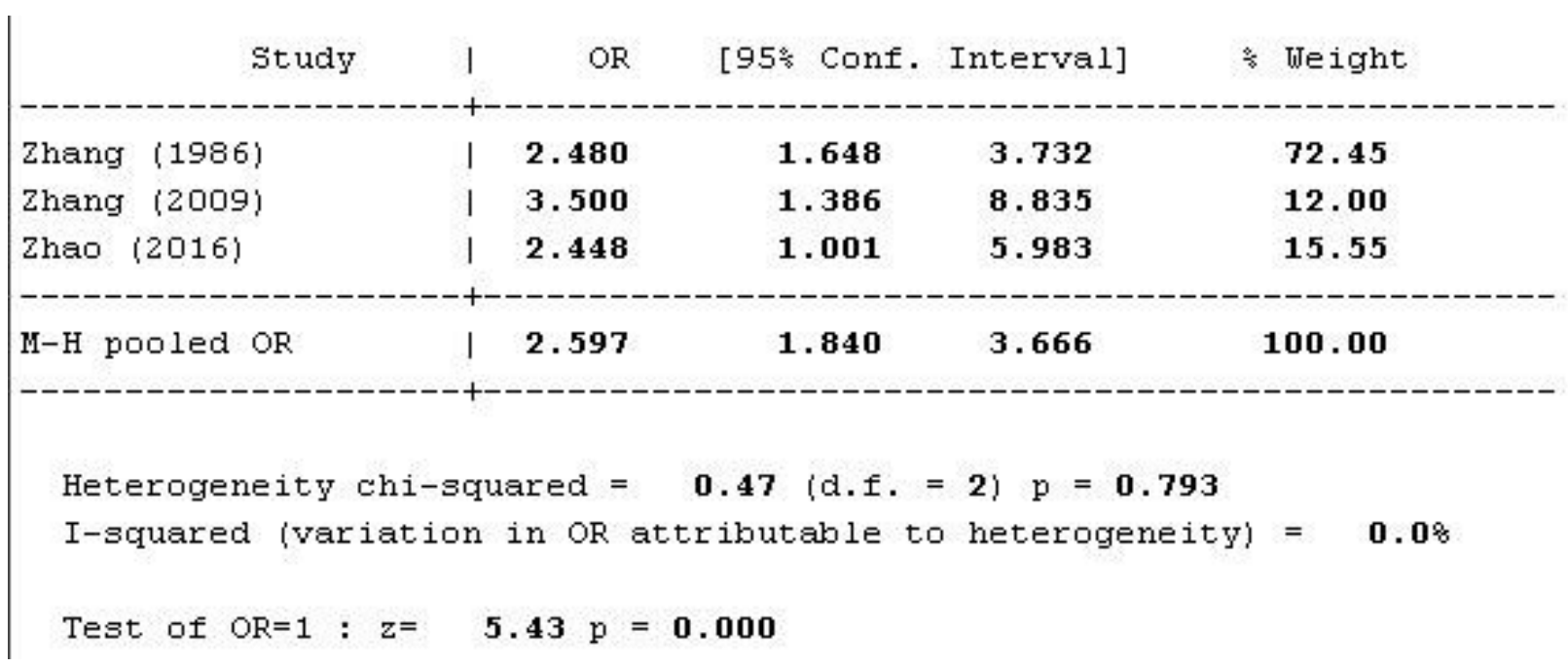

Figure 2. Overall media use with presence or absence of childhood ADHD

\subsection{Assessment of heterogeneity and sensitivity analysis}

According to Figure 3, the pooled OR was 2.597 (95\% CI 1.84-3.67) with no significant heterogeneity (P $=0.793, \mathrm{I}^{2}=0 \%$ ), which means that there was no significant difference observed across all three studies. Influence analysis via the leave-one-out method was used to examine potential outliers and influential cases. No outliers and influential cases were found as shown in Figure 4. 
STUDY

ID

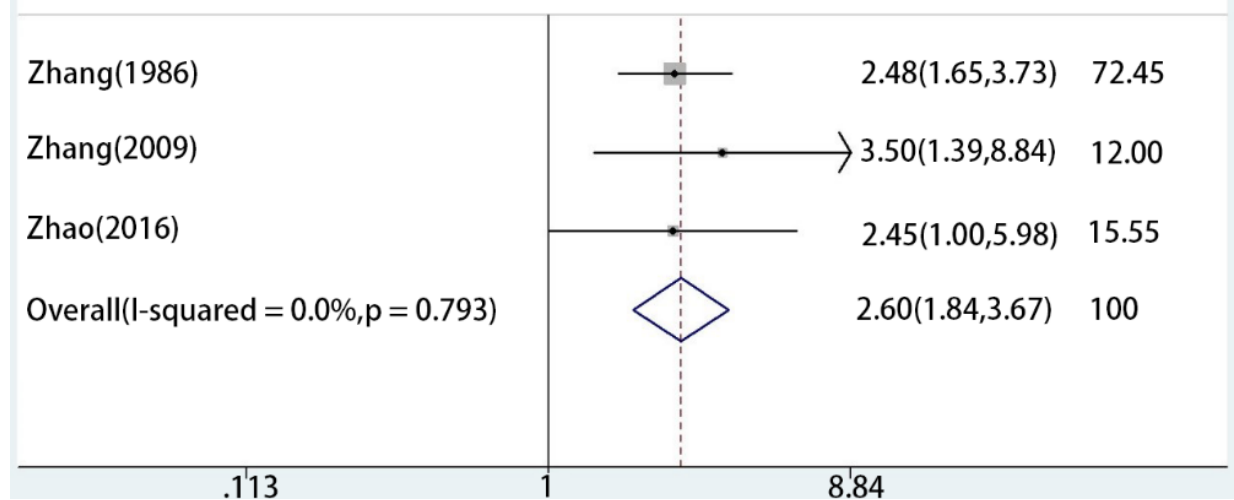

Figure 3. Heterogeneity among studies

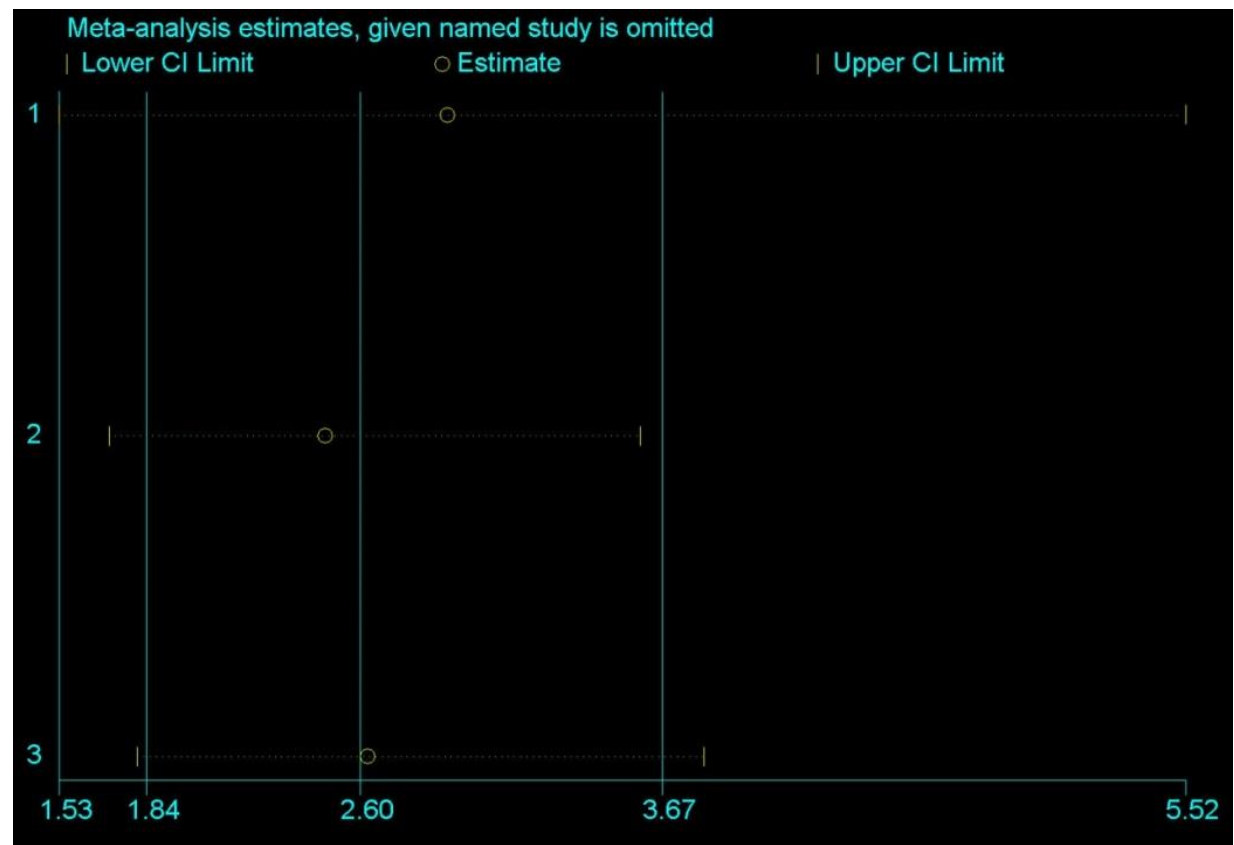

Figure 4. Influence analysis by leave-one-out method

\subsection{Publication bias}

In order to calculate the publication bias, gen $\log \mathrm{OR}=\log (\mathrm{ES})$ was used to generate the $\log \mathrm{OR}$ value. The publication bias of the literatures was evaluated by Begg's funnel plot (Figue 5(a)) with Begg's and Egger's tests (Figure 5(b)). There is no publication bias for the overall media use in these three studies. 

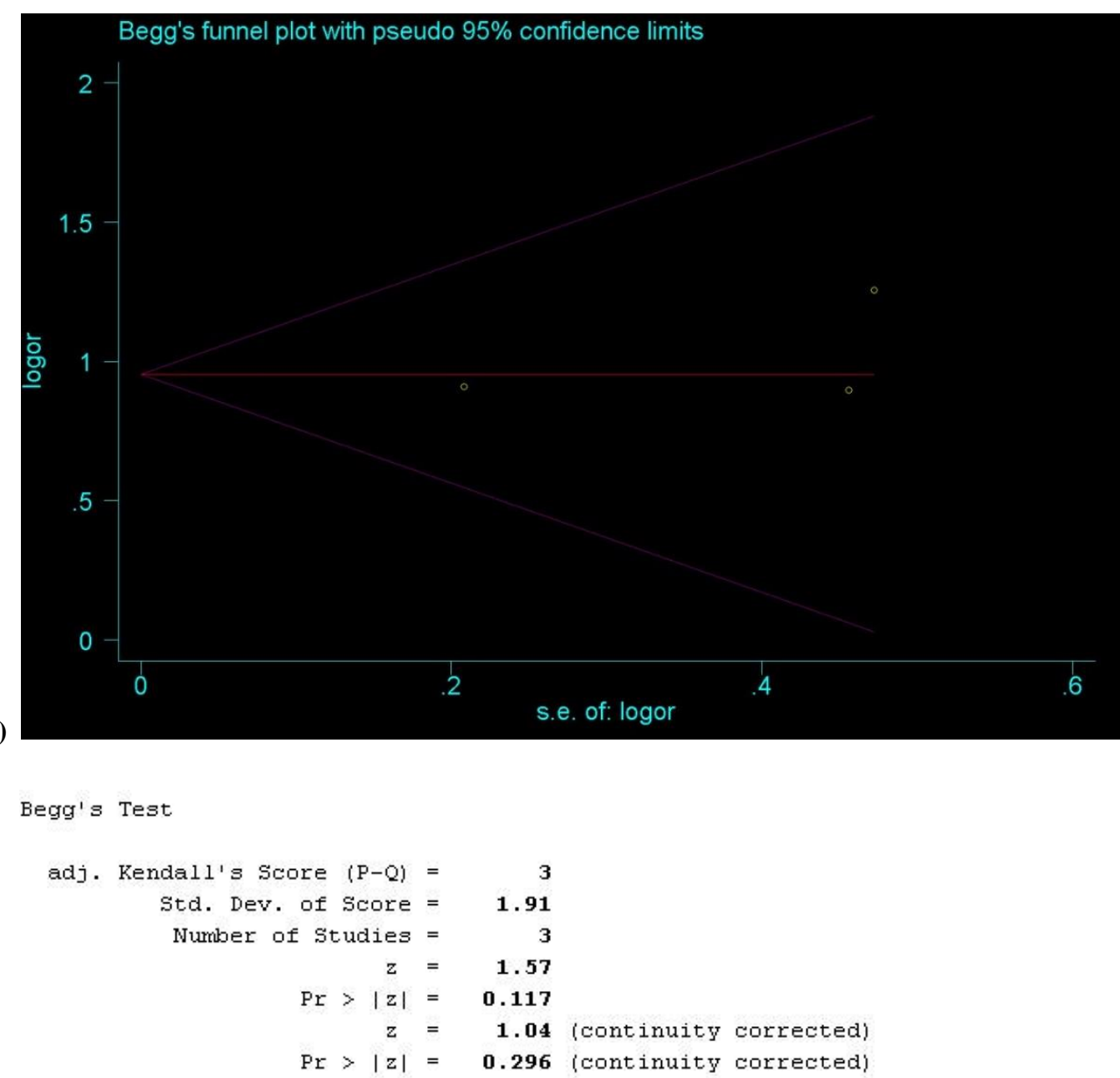

Egger's test

\begin{tabular}{r|rrrrrr}
\hline Std_Eff & Coef. & Std. Err. & t & P>|t| & [95\% Conf. Interval] \\
\hline slope & $\mathbf{. 7 6 9 1 5 4 1}$ & $\mathbf{. 2 4 5 2 4 4 8}$ & $\mathbf{3 . 1 4}$ & $\mathbf{0 . 1 9 6}$ & $\mathbf{- 2 . 3 4 6 9 7 7}$ & $\mathbf{3 . 8 8 5 2 8 5}$ \\
bias & $\mathbf{. 6 5 5 2 9 2 7}$ & $\mathbf{. 8 0 4 3 7 8 5}$ & $\mathbf{0 . 8 1}$ & $\mathbf{0 . 5 6 5}$ & $\mathbf{- 9 . 5 6 5 3 0 5}$ & $\mathbf{1 0 . 8 7 5 8 9}$
\end{tabular}

(b)

Figure 5. Publication bias by (a) Begg's funnel plot with (b) Begg's test and Egger's test

\section{Discussion}

In this meta-analysis, the aim was to summarize the findings in empirical literatures on the relationship between overall media use and ADHD-related behaviors in children and adolescents. The relationship between overall media use and ADHD was analyzed based on Chinese children.

Only three studies used the measure of overall media use and ADHD symptoms in children.

For all three studies, the OR was positive, reflecting that overall media use will cause ADHD. The results were similar to those of Nikkelen's ${ }^{[15-17]}$.

Due to the lack of data, subgroup research, such as comparing age and sex, cannot be performed.

Moreover, the results for these outcomes should be interpreted with caution because of the limited number of studies and also in view of no heterogeneity across the studies; as known, individual studies differ greatly in their study designs and samples (i.e., children's age and sex distribution). 


\section{Limitation}

Similar with any meta-analysis, this meta-analysis also has some limitations. First, this meta-analysis only included published studies, it is inevitable that an observed effect might suffer from publication bias because studies with null results tend not to be published. Other than that, this meta-analysis only included cross-sectional studies where causal inference is a real concern, and the time of media use was self-reported which may have led to measurement error.

\section{Conclusion}

In this meta-analysis, it was found that there is a positive relationship between overall media use and ADHD-related behaviors. More importantly, this meta-analysis clearly revealed a stronger combined OR value $(\mathrm{OR}=2.597)$ than in Lingineni's research in the United Stated $(\mathrm{OR}=1.32)^{[18]}$. It appears that Chinese children are more susceptible to media influence than American children. To conclude the adjustment, there is a vital need for empirical research on more samples.

\section{Funding}

This research was supported by Guangdong Social Science Fund (GD20XXW06) and Zhuhai Social Science Fund (2021YBB077).

\section{Disclosure statement}

The authors declare that there is no conflict of interest.

\section{References}

[1] Tong L, Shi H, Zang J, 2013, Prevalence of ADHD in Children of China: A Systematic Review and Meta Analysis. Chinese Journal of Public Health, 2013(09): 1279-1283.

[2] Liang D, Wang Y, Li C, 2009, Epidemiological Survey of Attention Deficit Hyperactivity Disorder Among Students Aged 6 To 18 Years in the Central District of Changchun. Maternal and Child Health Care of China, 2009(04): 522-524.

[3] Sun D, Yi M, Li Y, 2009, Status of Attention Deficit Hyperactivity Disorder Among Children Aged 16 to 6 Years in Binzhou City, Shandong. Chinese Mental Health Journal, 2009(11): 806-809.

[4] Zhao R, 2013, The Etiological Factors and Treatment of Children with Attention Deficit Hyperactivity Disorder. Chinese Journal of Child Health Care, 2013(06): 620-622.

[5] Rideout VJ, Foehr UG, Roberts DF, et al., 2010, Generation M: Media in the Lives of 8- to 18-YearOlds, Kaiser Family Foundation, Menlo Park, California.

[6] Zimmerman FJ, Christakis DA, Meltzoff AN, 2007, Associations between Media Viewing and Language Development in Children Under Age 2 Years. J Pediatr, 151(4): 364-368. DOI: 10.1016/j.jpeds.2007.04.071

[7] Swing EL, Gentile DA, Anderson CA, et al., 2010, Television and Video Game Exposure and the Development of Attention Problems. Pediatrics, 126(2): 214-221. DOI: 10.1542/peds.2009-1508

[8] Ye Y, Zhou Y, Chen K, 2009, Investigation on the Status of DVD/VCD Exposure in Infants and Young Children in Wenzhou City. Chinese Preventive Medicine, 2009(12): 1060-1064.

[9] Christakis DA, 2009, The Effects of Infant Media Usage - What Do We Know and What Should We Learn. Acta Pædiatrica, 98(1): 8-16. 
[10] Zimmerman FJ, Christakis DA, 2007, Associations between Content Types of Early Media Exposure and Subsequent Attentional Problems. Pediatrics, 120(5): 986-992. DOI: 10.1542/peds.2006-3322

[11] Levine LE, Waite BM, 2000, Television Viewing and Attentional Abilities in Fourth and Fifth Grade Children. Journal of Applied Developmental Psychology, 21(6): 667-679.

[12] Kronenberger WG, Mathews VP, Dunn DW, et al., 2005, Media Violence Exposure and Executive Functioning in Aggressive and Control Adolescents. J Clin Psychol, 61(6): 725-737. DOI: $10.1002 /$ jclp.20022

[13] Gao M, Wen F, Zhou K, et al., 2012, Analysis on the Influencing Factors of Attention Deficit Hyperactivity Disorder in Children Aged 7 to 14 Years in Shenzhen City. Journal of Applied Clinical Pediatrics, 2012(12): 922-924.

[14] Lin W, Tang C, 1985, Family Environment, Attention Behavior and the Secretory Pattern of Peripheral Adrenaline in Children with ADHD. Acta Psychologica Sinica, 1985(04): 410-417.

[15] Nikkelen SW, Valkenburg PM, Huizinga M, et al., 2014, Media Use and ADHD-Related Behaviors in Children and Adolescents: A Meta-Analysis. Dev Psychol, 50(9): 2228-2241. DOI: 10.1037/a0037318

[16] Nikkelen SWC, Vossen HGM, Valkenburg PM, 2015, Children's Television Viewing and ADHDrelated Behaviors: Evidence from the Netherlands. Journal of Children and Media, 9(4): 399-418. DOI: 10.1080/17482798.2015.1088872

[17] Nikkelen SWC, Vossen HGM, Valkenburg PM, et al., 2014, Media Violence and Children's ADHDRelated Behaviors: A Genetic Susceptibility Perspective. Journal of Communication, 64(1): 42-60. DOI: $10.1111 /$ jcom.12073

[18] Lingineni RK, Biswas S, Ahmad N, et al., 2012, Factors Associated with Attention DeficitHyperactivity Disorder Among US Children - Results from a National Survey. BMC Pediatrics, 12: 50. 